\title{
Merging Topics in Well-Formed XML Topic Maps
}

\author{
Richard Widhalm and Thomas A. Mueck \\ University Of Vienna \\ Institute for Computer Science and Business Informatics \\ Rathausstr. 19 \\ 1010 Vienna, Austria \\ rwidhalm@gmx . at \\ mueck@ifs.univie.ac.at
}

\begin{abstract}
Topic Maps are a standardized modelling approach for the semantic annotation and description of WWW resources. They enable an improved search and navigational access on information objects stored in semi-structured information spaces like the WWW. However, the according standards ISO 13250 and XTM (XML Topic Maps) lack formal semantics, several questions concerning e.g. subclassing, inheritance or merging of topics are left open. The proposed TMUML meta model, directly derived from the well known UML meta model, is a meta model for Topic Maps which enables semantic constraints to be formulated in OCL (object constraint language) in order to answer such open questions and overcome possible inconsistencies in Topic Map repositories. We will examine the XTM merging conditions and show, in several examples, how the TMUML meta model enables semantic constraints for Topic Map merging to be formulated in OCL. Finally, we will show how the TM validation process, i.e., checking if a Topic Map is well formed, includes our merging conditions.
\end{abstract}

\section{Introduction}

The Topic Maps standard ISO 13250 7] as well as XTM (XML Topic Maps) [12] provide for the semantic characterization of information objects across the WWW or company-controlled intranet or extranet platforms. They externally describe the underlying information objects (documents, web pages, etc.) without changing them. With Topic Maps, which are syntactically based on XML (or SGML for ISO13250), semantic networks consisting of topics and their relationships can be built, thus enhancing the flexibility of search queries and navigational access on the underlying information objects. They can be used as a flexible, generic index for knowledge bases. In a topic map, every real world subject (equal if a WWW resource or an abstract thing) is represented by a topic. Each topic may be an instance of several topic types (which are also topics), may have several lexical names in different scopes (where a scope is the area where the according name for the topic is said to be valid and each scope

D. Fensel et al. (Eds.): ISWC 2003, LNCS 2870, pp. 6479 2003.

(C) Springer-Verlag Berlin Heidelberg 2003 
is described by a set of topics) and may show several occurrences in different WWW resources. An occurrence is a link, typed by a topic, to an information object. Topics can be interrelated via n-ary associations, where each topic plays a certain role which is again expressed by another topic. Associations can be typed via a topic. Further, it is possible to create generalization hierarchies for topic types, association types and occurrence types. Occurrences, names and associations can be placed within a scope. Each topic T has an identificator, which can be a WWW resource or another topic, indicating or itself being the subject for which $\mathrm{T}$ stands. Furthermore, Topic Maps define two topics $T_{1}$ and $T_{2}$ as identical, if they are identified by the same resource or if they both exhibit the same name $\mathrm{N}$ in the same scope $\mathrm{S}$. In that case, they shall be merged to a single topic bearing all the characteristics of the original topics. In this paper, we will focus on the issue of merging topics. We will show how the TMUML meta model lets one formulate the actual XTM merging conditions with the OCL (object constraint language), defined within the UML specification [9]. We will also show specific circumstances under which merging would not be desirable, although the XTM criteria would be met. Therefore, we will inspect the consequences of the merging of two topics of a different kind (like an association type merged with an occurrence type). Consequently, we present suggestions for additional OCL constraints showing how the TMUML meta model can help to overcome such issues. Of course, the presented constraints are only considered as suggestions that may further be extended or only partially applied according to specific needs. The following section briefly introduces the TMUML meta model, succeeded by an overview about related work. Afterwards, we will describe the synonymy relation used for merging in the TMUML meta model. Chapter 5 expresses the XTM merging rules in OCL and adds a further, implicated merging rule. Afterwards, we will inspect situations of merging different kinds of topics (like association types, occurrence types, topic types or topic instances) and show the processing steps within our XTM validation and the functionality of the OCL checker. The conclusion will point out the meaningfulness of merging in largely designed Topic Maps systems and give a prospect on our further work.

\section{Topic Maps and the TMUML Meta Model}

In [19] and [20], the TMUML meta model has been presented, including a detailed description as well as the according class diagrams. It is a meta model for Topic Maps, directly derived from the UML meta model. It does not make use of all of the UML meta model components, only those which are also relevant for Topic Maps (like Association or GeneralizableElement). Note that topics are divided into the meta classes TopicType (for topics used as types for other topics), TopicObject (topics not functioning as types but as instances of types), AssociationTopicType (similar to the UML AssociationClass, representing types for association instances) and OccurrenceType (types for occurrences). The TopicAssociationEnd defines, like the AssociationEnd in UML, the allowed TopicType for a role within the according TopicAssociation, and also 


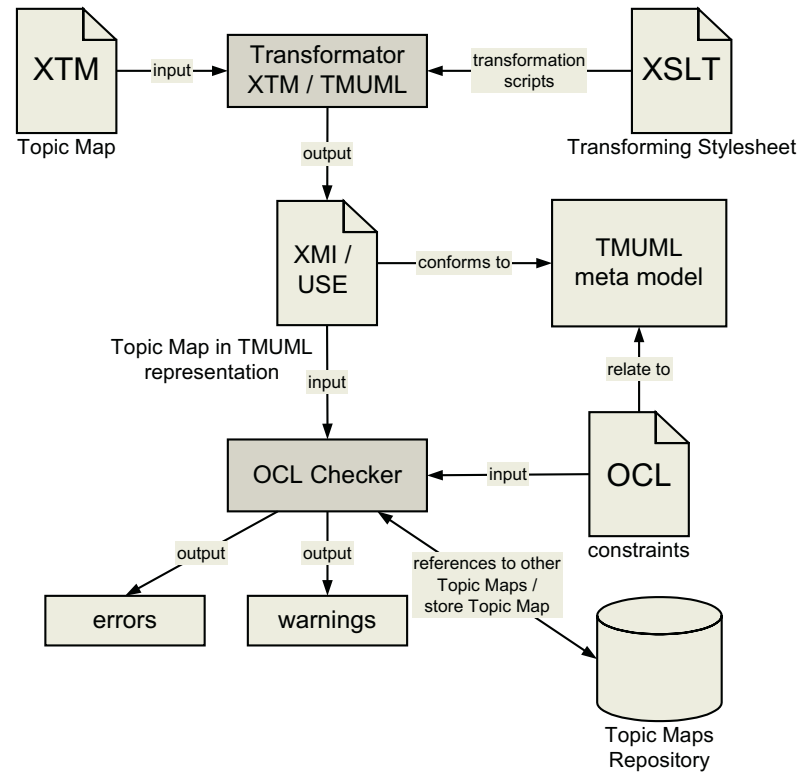

Fig. 1. Topic Maps validation process

its multiplicity. Base names and its subvariants are all represented by TopicName (which may be part of a name hierarchy). See [19] and 20] for a detailed description of the TMUML meta model. The TMUML meta model enables our process for validating the well-formedness of a topic map. Fig. 1 schematically shows our approach in the context of validating an XTM topic map before storing it within a repository.

First, the XTM topic map is transformed into an UML representation. For the transformation, XSLT would be an appropriate method, while for the representation, the XMI metadata interchange format [10] or the USE syntax (see [5]) could be used. While the first alternative provides more openness, the second will fit perfectly if using the USE tool for validating OCL constraints. The OCL checker is the component which is responsible for validating the UML representation of the topic map against the OCL constraints that make up well-formedness. For our testing purposes, we used the USE tool, but any other tool capable of OCL validation may be used instead. The OCL checker is then responsible for generating appropriate error and warning messages. After proving a topic map to be valid, it can be inserted into a repository. Also, reading repository data may be important for the OCL checker, in case topics could be merged according to the name based merging principle (see section [5).

The following example shows the definition of a simple OCL constraint, stating that no Topic shall be identified by itself.

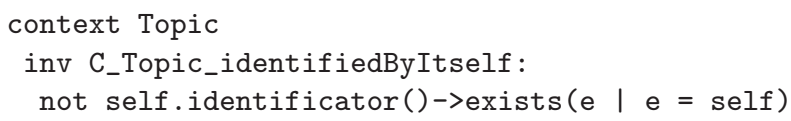


It makes use of the operation identificator(), which is defined in the context of Topic and retrieves all Identificators that are related to the context Topic via an Identification relationship in the TMUML meta model. An Identificator may be a subject constituting resource, a subject indicating resource or another topic (see [12]).

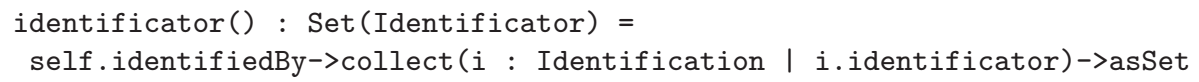

\section{Related Work}

An early work on constraining Topic Maps is [14, where several suggestions for constraints at the instance layer of a topic map are given (without a formal method). An overview about the idea behind constraints for Topic Maps in general can be found in [6]. In [1], Ontopia describes their own solution for a Topic Maps constraint language, called OSL (Ontopia Schema Language). It allows for a more constrained and precisely defined Topic Maps schema definition, but again is not usable for constraining the Topic Maps meta model. With OSL, the types and roles allowed within certain association types may be specified, but, for example, the effects of subclassing association types in general are not treated (the semantics of inheritance). Our method is best suitable for solving the second kind of problem, while it may also be used for the first kind. A similar approach is sketched by the TMCL 13, the Topic Maps Constraint Language, although it has not yet surpassed the level of a requirements suggestion. 2] presents a formal model for Topic Maps, consisting only of topics and associations, which are contained in a homogenous hypergraph. Meta associations like instancing, subclassing or scoping are contained in a shift hypergraph, that compounds components of the homogenous hypergraph and thus establishes semantic layers (e.g. separating role types, topic types or association types). Although this approach applies formal semantics to Topic Maps using hypergraphs, it does not mention additional constraints on the Topic Maps meta model or how they can be applied. Our approach goes beyond that and provides means for the automation of semantic constraints using OCL and USE (or comparable OCL tools). Moreover, the hypergraph model does only distinguish between topics and associations, while names, occurrences or resources have to be modelled by topics. Actually, names have different properties compared to topics in the Topic Maps meta model. Names can not be merged or have occurrences or have names etc. Further, there may be difficulties due to the fact that the semantics of the associations in the shift hypergraph (denoted as $\theta$ ) is only determined by the semantic layers of the connected topics. For example, there may be different meanings of connections from a role type to a topic type, one may mean a "subclass" relation, the other may mean a type constraint for the topic members playing the role type in the according association type.

Additional information on Topic Maps can be found in [8], [18, [1] and [3]. The first gives an introduction to the fundamental concepts of Topic Maps and introduces a system architecture for applications based on a distributed 


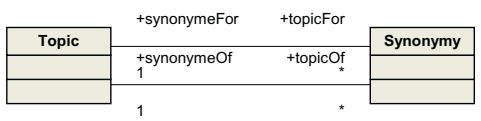

Fig. 2. The Synonymy relation

Topic Maps repository. The second describes a webbased search engine based on a Topic Maps repository, from the conceptual approaches up to a pseudocode, including a prototypic query language. The third publication discusses a range of possible application areas for Topic Maps, while the fourth presents preliminary guidelines to the design of certain topic maps. Detailed information on OCL can be found in the according specification [9] and in [17]. In [4], uncertainties in the semi-formal OCL specification are discussed. In [16], a formal semantics for the OCL is introduced.

\section{The Synonymy Relation}

XTM defines that two topics $T_{1}$ and $T_{2}$ shall be merged to one single topic $T_{3}$, that inherits all characteristics of $T_{1}$ and $T_{2}$ (names, occurrences, association memberships, types), if $T_{1}$ and $T_{2}$ meet some merging criteria and can therefore be deemed to reify (stand for) the same subject. Unfortunately, this would mean that they could not be separated from each other. This should be possible in the context of a Topic Maps repository: in the case one wants to remove the topic map $T M_{1}$, which contains $T_{1}, T_{2}$ which belongs to the topic map $T M_{2}$ should remain in the repository, in the state it was before the merging with $T_{1}$. For this reason, we will keep topics which shall be merged separated in the TMUML meta model, but interrelate them via a Synonymy relationship, an equivalence relationship which we have to introduce in our TMUML meta model. Fig. 2 shows the additional class Synonymy and its associations.

When removing a previously merged Topic $\mathrm{T}$, the topics which have been merged with $\mathrm{T}$ can still remain in the repository, only their Synonymy link to $\mathrm{T}$ will be removed.

In our set of constraints, every time we refer to a Topic T or its characteristics, we also have to refer to the characteristics of all the Topics $T S Y N_{i}, i=1, \ldots, n$, which are directly or transitively connected to $\mathrm{T}$ via a Synonymy relation. We do this by providing an OCL operation called closure() yielding the transitive closure of a Topic T, formally $\left\{T \cup\left\{T S Y N_{i} \mid T S Y N_{i}\right.\right.$ is (transitively) synonymous to $\left.\left.T ; i=1, \ldots, n\right\}\right\}$.

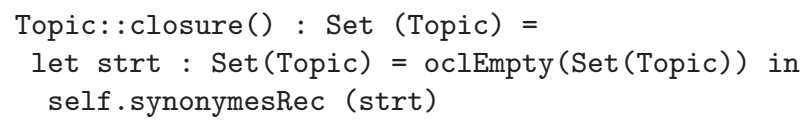

strt denotes a local variable of the type Set(Topic), initialized with an empty set of Topics. synonymesRec is an operation in the context of Topic that takes a set $\mathrm{S}$ of Topics as argument and returns for a Topic $\mathrm{T}$ a set of Topics 
containing $\mathrm{T}$ and the Topics returned by recursively calling synonymesRec (which takes $\mathrm{S}$ extended by $\mathrm{T}$ ) for all Topics directly connected to $\mathrm{T}$ via the Synonymy relation.

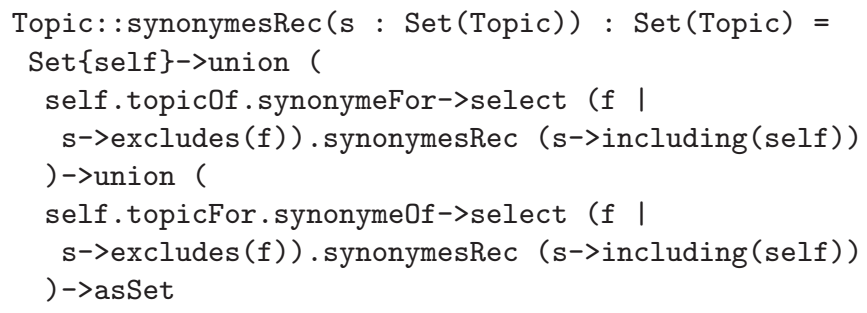

Thus, by calling $\mathrm{T} . \mathrm{closure}()$, we receive $\mathrm{T}$ and all other Topics that are synonymous to T. Further, to determine whether two Topics are equal, we can not simply compare the two Topics, we have to find out if one is contained within the closure of the other. We therefore provide the operation Topic: : equals(), which is defined as follows.

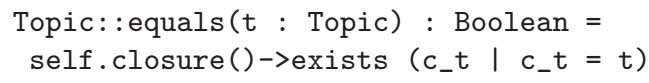

\section{$5 \quad$ Merging Conditions}

XTM defines two merging conditions. The naming constraint-based merge [12] says that two Topics $T_{1}$ and $T_{2}$ have to be merged if $T_{1}$ has a base name $N_{1}$ in the scope $S_{1}$, and $T_{2}$ has a base name $N_{2}$ in scope $S_{2}$, and $N_{1}$ and $N_{2}$ are equal strings and $S_{1}$ and $S_{2}$ are equal sets of Topics. We define this condition as an OCL operation called Topic: : nameBasedMerge (b: Topic), which checks whether the context Topic may be merged with b due to the naming constraintbased merge.

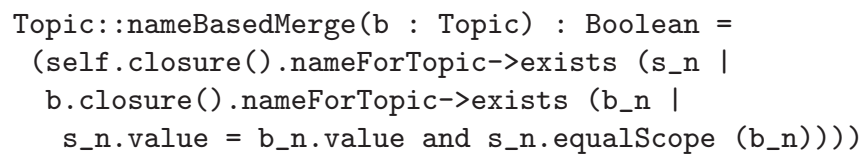

Here, ScopableElement: :equalScope ( $s$ : ScopableElement) is used to compare the scopes of two ScopableElements, which are names in our case (Occurrences and Associations are also ScopableElements. It uses two other operations, which are also defined within the context of ScopableElement.

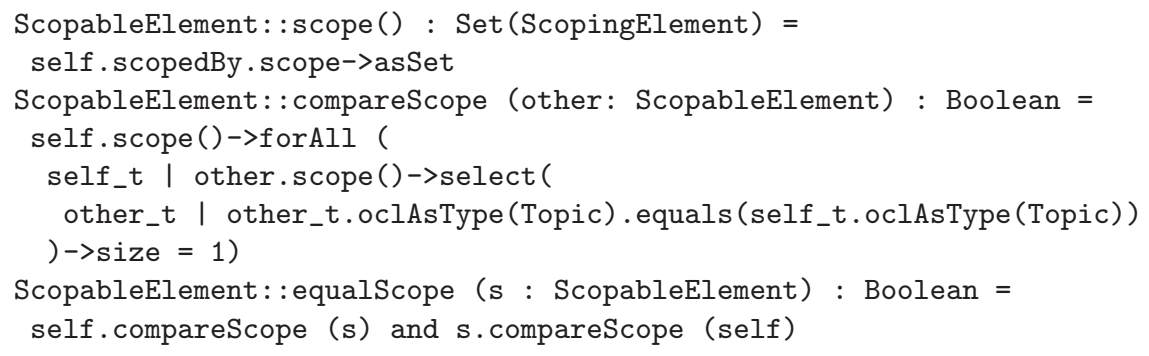


Note that Topic.nameForTopic yields all TopicNames for a Topic, TopicName.value is the lexical represenation of a name, and ScopableElement. scope() yields the set of Topics (which are ScopingElements) making up the scope of a ScopableElement.

The other XTM merging condition is called subject-based merge 12 and says that two Topics $T_{1}$ and $T_{2}$ have to be merged if $T_{1}$ has an Identificator $I_{1}$, and $T_{2}$ an Identificator $I_{2}$, where the URIs of $I_{1}$ and $I_{2}$ are equal or $T_{1}=I_{2}$ or $T_{2}=I_{1}$. Note that also in this operation we have to excessively use Topic: : closure().

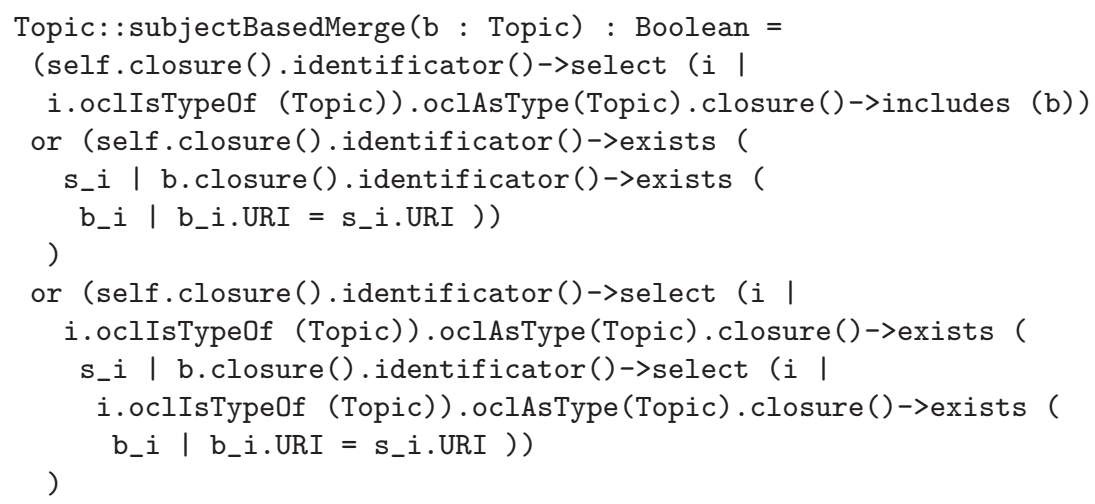

Further, note that the determination of URI equality is not discussed in this paper and therefore, for the time being, simplified by comparing the URI strings. Instead, the information objects that can be reached by the specific URIs should be compared, and also time (timestamps) may play an important role. See also 15] for more details on URIs.

We will call two Topics $T_{1}$ and $T_{2}$, which meet one of the two XTM merging conditions, candidates for merging, as they should be merged according to XTM, but this merging and its consequences has to be examined and possibly prohibited due to some resulting complications.

A situation where merging is still not possible, even if one of the aforementioned conditions would be met, is, when the two candidate Topics $T_{1}$ and $T_{2}$ both have a SubjectConstitutingResource (which means, the resource is the subject itself) as Identificator - call them $S C R_{1}$ and $S C R_{2}$ - and $S C R_{1}$ is different from $S C R_{2}$ (they have different URIs). In XTM, only one SubjectConstitutingResource is possible for a Topic, which is quite intuitive. Fig. [3 shows this issue schematically. There, $T_{1}$ is synonymous to $T_{2}$ due to having an equal base name in the same scope $\left\{T_{s}\right\}$. Because they both exhibit a SubjectConstitutingResource as Identificator, differing from each other, the OCL checker has to raise an error.

We have to check this situation in our validation process and therefore suggest an appropriate operation called Topic: :noDifferentSCR (b:Topic). It uses an operation called Topic: :ownSCRIdentificators(), which is defined in the following. 


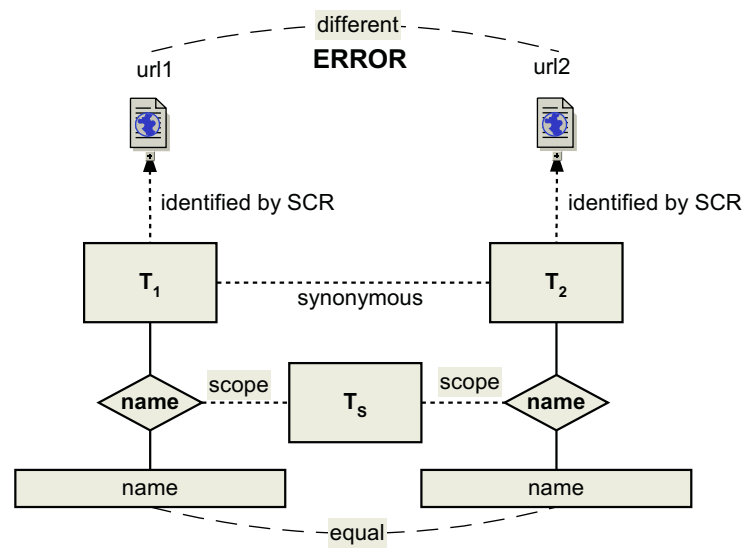

Fig. 3. Different SubjectConstitutingResources as Identificator

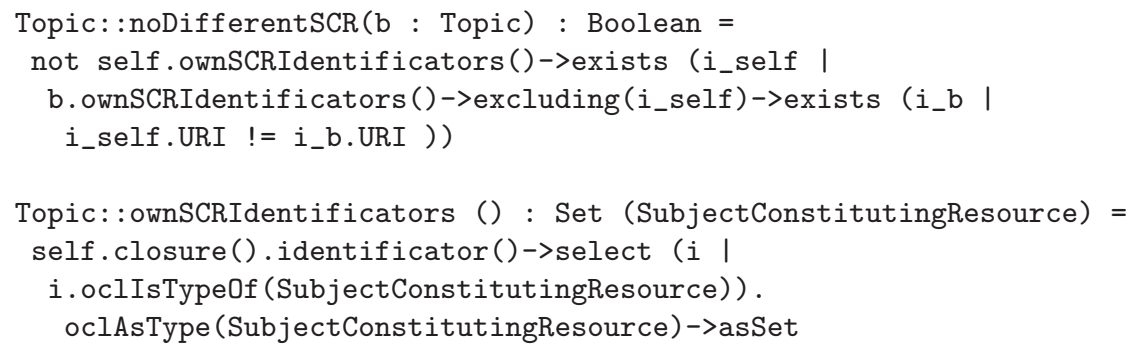

\section{Merging Different Kinds of Topics}

What is yet unconsidered in the merging criteria is the fact that the two Topics $T_{1}$ and $T_{2}$, which are candidates for merging due to one of the merging conditions, can be of a different kind. The possible kinds are: TopicType (TT), TopicObject (TO), AssociationTopicType (AT) and OccurrenceType (OT). We will use the abbreviations (in brackets) for the four kinds of Topics.

In the following, we will examine many of the possible combinations (refer to our further work for a full description of all combinations, including an extensive description of the combination AT - AT) and find out for any combination, if merging shall be prohibited due to the possibility of a semantic inconsistence or not.

\subsection{TT - TT, TO - TO, OT - OT}

First, we will examine the merging of two TopicTypes $T T_{1}$ and $T T_{2}$. A TopicType $T T_{1}$ may define a TopicAssociationEnd of an AT, where the multiplicity_min (the lower bound of the multiplicity) is greater than 0. Look at the example in fig. 4 Some constraint, which will not be shown in this work 


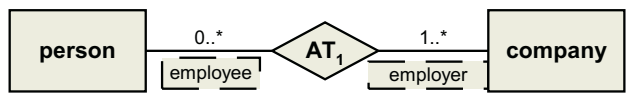

Fig. 4. Example for a Multiplicity Constraint on an AT

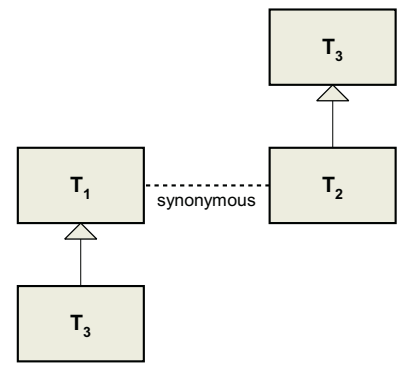

Fig. 5. Circular Inheritance After Merging

due to space reasons, will have to guarantee that every instance of "person" is connected to at least one company via a TopicAssociation of type $A T_{1}$. Thus, when merging the TopicType "person" with some other TopicType $T_{2}$, all instances of $T_{2}$ will also have to have at least one TopicAssociation of type $A T_{1}$. We will call this the MultiplicityMinProblem. This can be realized either by formulating an operation that does this check before merging (called from inside the preconditions of the merge operation), or by an invariant that has to hold any time and yields false after merging.

A further problem may arise when $T T_{1}$ and $T T_{2}$, which are candidates for merging, define the roles of the two TopicAssociationEnds $T A E_{1}$ and $T A E_{2}$ of the same AT. When desiring unique roles within the same AT, the appropriate OCL invariant will yield false after merging $T T_{1}$ and $T T_{2}$. We will call this the UniqueRolesProblem. Finally, the CircularInheritanceProblem may arise after merging, so that $T T_{1}$ and $T T_{2}$ are then in a circular generalization relationship chain. Fig. 5 shows this schematically.

A circular inheritance prohibiting constraint, like the one adopted by the TMUML meta model from the UML GeneralizableElement (constraint 3 for GeneralizableElement in [9]), would yield false after merging. Again, these problems can also be checked by OCL operations before performing the merge operation.

By merging two TopicObjects $T O_{1}$ and $\mathrm{TO}_{2}, \mathrm{TO}_{1}$ is afterwards instance of its own TTs and the TTs of $\mathrm{TO}_{2}$. The MultiplicityMinProblem, as described in the previous section, may also arise here. TopicObjects may also define the role of some property of an AT, so in this case, the UniqueRolesProblem also may arise. 


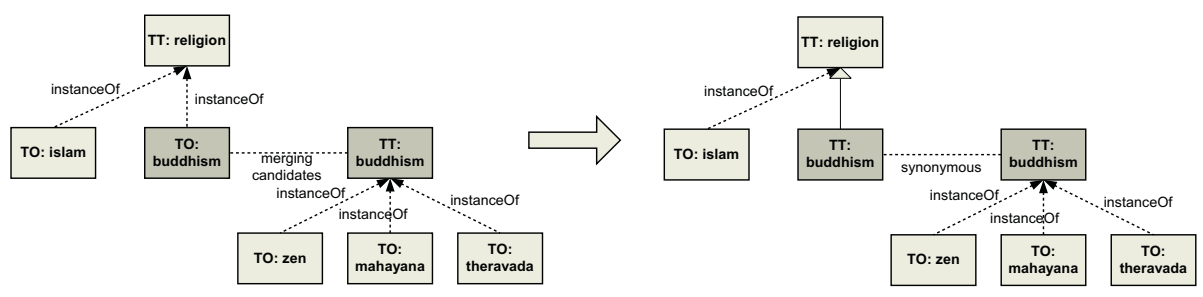

Fig. 6. Merging TT - TO

At the moment, there is no mechanism to constrain an OT to be only applicable to several TTs (like the TopicAssociationEnds do for ATs). Therefore, the merging of two OccurrenceTypes $O T_{1}$ and $O T_{2}$ should be no problem.

\subsection{TT - TO, AT - TO, OT - TO}

When merging two Topics of a different kind, the question arises whether the resulting Topics should be of the one kind or of the other. Similarly, when establishing a Synonymy relation in our TMUML meta model for two different kinds of Topics, we have to agree upon one kind for both Topics. In the case of merging $T T_{1}$ with $T O_{2}$, it is not possible to change $T T_{1}$ to $T O_{1}$, because $T T_{1}$ could have subtypes and may be used in TopicAssociationEnds as type constraints. But changing $\mathrm{TO}_{2}$ to $T \mathrm{~T}_{2}$ would be an appropriate measurement, requiring every class-instance relation from $\mathrm{TO}_{2}$ to some $T T_{2 \text { super }}$ to be transformed by the merging operation to a subtype relation from the resulting $T T_{2}$ to the original $T T_{2 \text { super. }}$ This works, because a $\mathrm{TO}$ does not have any further capabilities in the TMUML meta model than a TT has, and a TT may also play a role within an association instance (see also [19]). Fig. 6 shows an example. There, the Topics "TT:buddhism" and "TO:buddhism" are candidates for merging. "TO:buddhism" has to be converted to "TT:buddhism", and the class-instance relation between "TO:buddhism" and "TT:religion" is converted to a subtype relation.

Note that also in the TT-TO case, a UniqueRolesProblem may occur after merging. The following OCL operation is called Topic: : changeToTT ( $t$ : TopicType), where the context Topic is the TO, $t$ is the TT and TopicObject. _class is a TMUML relation to the TTs of the context TO.

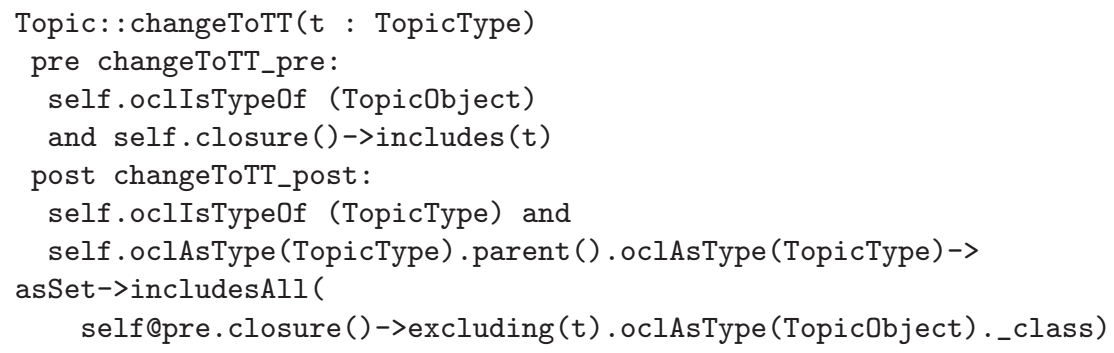


After calling this operation after the merge operation, which already set the Synonymy relation between the TO and TT, no Topic: :closure() contains both a TT and a TO. Note that this strategy is a suggestion - of course, other strategies can be realized with OCL constraints. The merging of a TT and TO could in general be prohibited, which would be a more performant, but also a more restrictive solution. The two cases of merging an AT with a TO or an OT with a TO can be treated similar to the TT - TO case. As a TO has no instances and no more capabilities in the TMUML meta model than an AT, the TO can be converted to and AT after merging the TO and the AT. Note that an AT can also be a member of a TopicAssociationEndInstance (see [19]). The same is true for an OT and a TO. For space reasons, the operations Topic: : changeToAT ( $t$ : AssociationTopicType) and Topic: : changeToOT(t : OccurrenceType) are not shown here.

\subsection{TT - AT, TT - OT}

Converting an $A T_{2}$ to a $T T_{2}$, when merging $T T_{1}$ and $A T_{2}$, will often be impossible, as $A T_{2}$ may have a supertype $A T_{2 \text { super }}$, and $T T_{1}$ cannot be subtype of an AT (a TT has no TopicAssociationEnds). Intuitively, in this situation we will try to specialize $T T_{1}$ and convert it to $A T_{1}$. As $\mathrm{AT}_{1}$.properties() will (after the conversion from a TT to an AT) obviously be empty, $A T_{1}$ simply will have the same properties as $A T_{2}$ after the merge (for the definition of AT.properties, which yields the TopicAssociationEnds of an AT, taking inheritance into account, see 19] or [20] ). On the other hand, $T T_{1}$ and all its subtypes must not have any instances (TOs), because this would mean these TOs should actually be TopicAssociations after the merge, which is not possible. Moreover, all supertypes of $T T_{1}$ are neither allowed to have any instances, because after converting $T T_{1}$ to $A T_{1}$, each direct or transitive supertype $T T_{x}$ of $A T_{1}$ has to be abstract (if not so, $T T_{x}$ would be a class containing both TopicObjects and TopicAssociations, which are fundamentally different constructs in Topic Maps). Thus, this kind of merging will rarely be possible, which seems intuitive, as it would mean a mixing of topics and associations, which are fundamentally different meta constructs within Topic Maps. Nevertheless, the following shows the formal representation of our suggested strategy, represented by the OCL operation Topic: : checkATTT (b : Topic), which yields true if the context Topic is a TopicType and all its supertypes and subtypes, which are also TopicTypes, have no instances. This operation is used inside the merge operation to determine the possibility of the merging of two candidate Topics. Note that allParents() recursively yields all supertypes of a TopicType, with respect to its closure, and allChildren() does so for its subtypes.

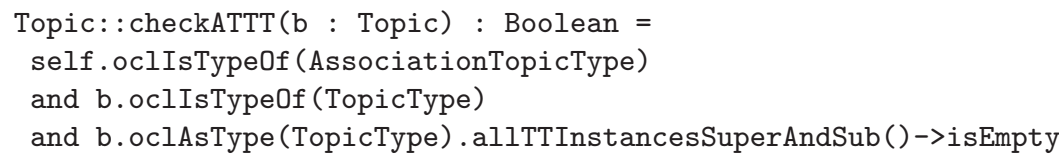

TopicType: :allTTInstancesSuperAndSub ()$:$ Set $($ TopicObject $)=$ self.allTTInstances()->union (self.allTTparentInstances()) 


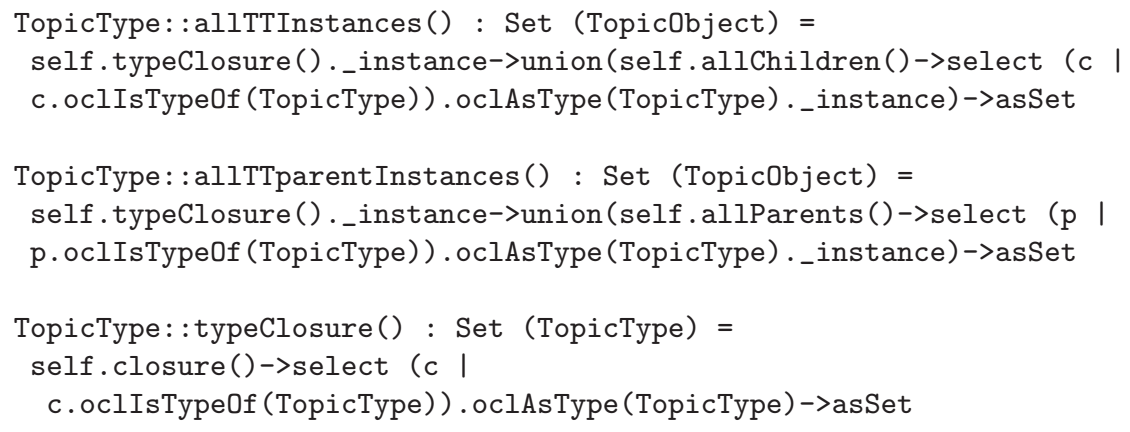

Note that TopicType._instance yields the set of direct instances (TOs) of the context TT.

\subsection{AT - OT}

Converting $A T_{1}$ to an $O T_{1}$ may only work in the case all supertypes and subtypes of $A T_{1}$, which are also ATs, have no TopicAssociationEnds, which would be a rather theoretical situation. Converting $O T_{2}$ to an $A T_{2}$ would only work, if $O T_{2}$ had neither instances (Occurrences) nor subtypes. Thus, we will simply prohibit merging of $A T_{1}$ and $O T_{2}$. At last, the OCL checker should report a warning that the merging of two candidate Topics was avoided.

\section{Merging Inside the OCL Checker}

In the previous sections, we have discussed the merging of different kinds of Topics and its effects on the consistency and well-formedness of a topic map and presented several additional constraints to avoid merging in certain situations. Finally, we will take a short look at the processing steps inside the OCL checker, which is not only responsible for checking all OCL invariants, it also has to perform merging by establishing the according Synonymy relations. Merging inside the OCL checker can be divided into the following three steps, where the set of processed topic maps may be, for example, all the topic maps within a repository and a new topic map to be stored into the repository:

For all Topics $T_{1}, T_{2}$ in the set of processed topic maps

1. check, if $T_{1}$ and $T_{2}$ are candidates for merging and - if they are of a different kind - if they can be merged

2. if $T_{1}$ and $T_{2}$ should be merged, establish a Synonymy relation between $T_{1}$ and $T_{2}$

3. if $T_{1}$ and $T_{2}$ should be merged, and $T_{1}$ is of a different kind than $T_{2}$, change the kind of $T_{1}$ or $T_{2}$ so that $T_{1}$ and $T_{2}$ are of the same kind

The core of the first step is the following operation Topic: : canMerge ( $b$ : Topics), which simply calls the already presented check operations. 


\section{Topic: : canMerge (b : Topic) : Boolean = (subjectBasedMerge(b) or nameBasedMerge(b)) and typeOfMergePossible(b) and noDifferentSCR(b)}

While the first two operation calls are for checking the XTM merging conditions, and the last one checks an additional condition implied by the XTM merging conditions, Topic: :typeOfMergePossible(b : Topics) encapsulates our suggested strategies for merging different kinds of Topics. It contains calls to Topic: :tOMP (b: Topic) in both directions, which is the actual application of our suggestions. There, operations like checkATTT() are called. Note that in this version, issues like the UniqueRolesProblem (section 6.1) are not checked in advance, merging is optimistically performed and may lead to an according constraint violation afterwards, which is reported by the OCL checker. Performing all checks before establishing the Synonymy relation, would call for additional OCL operations to be called from within tOMP().

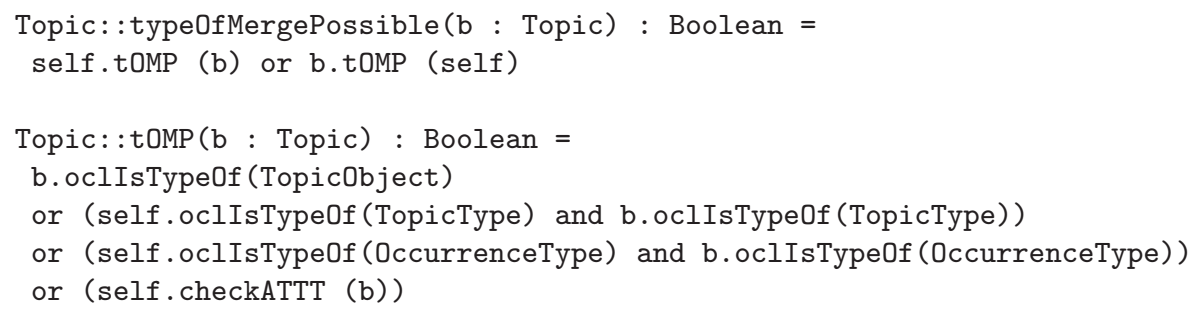

If $\mathrm{T}_{1}$.canMerge $\left(\mathrm{T}_{2}\right)$ yields true, the Synonymy relation can be established by $\mathrm{T}_{1}$.merge $\left(\mathrm{T}_{2}\right)$. This operation has to be implemented by an according method, in OCL we can only formulate its pre- and post-conditions.

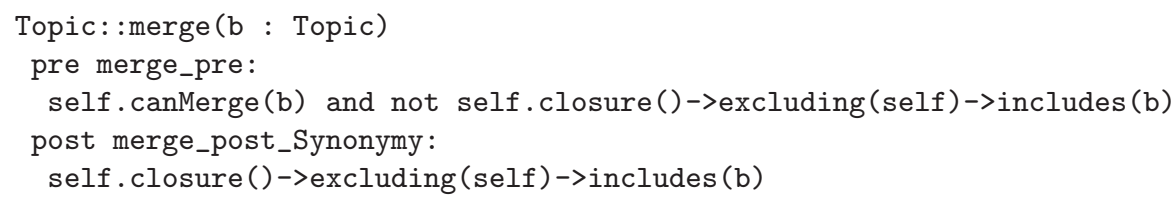

For the third step, a method Topic: :change (b : Topic) has to be implemented, which checks the kinds of $T_{1}$ and $T_{2}$ and subsequently calls the appropriate changeToXX() operation, if the kind of $T_{1}$ or $T_{2}$ has to be changed. One example for this would be the changeToTT (b: TopicType) operation presented in section 6.2. The postcondition of change() has to state that all Topics in the closure of the context Topic have to be of the same kind. We omit this for space reasons.

Fig. (7) schematically shows the processing steps of the OCL checker. At first, the previously introduced three merging steps are performed. Afterwards, all other constraints (OCL invariants for inheritance, instancing, etc.) are checked. If errors are to be reported, the OCL checker does so and stops further work, the topic map is considered not well-formed (and therefore can not be stored into a repository). Otherwise, if some merging has happened, the whole processing has to be repeated, as one merging may cause a further merging to happen. This situation is shown in fig. 8 


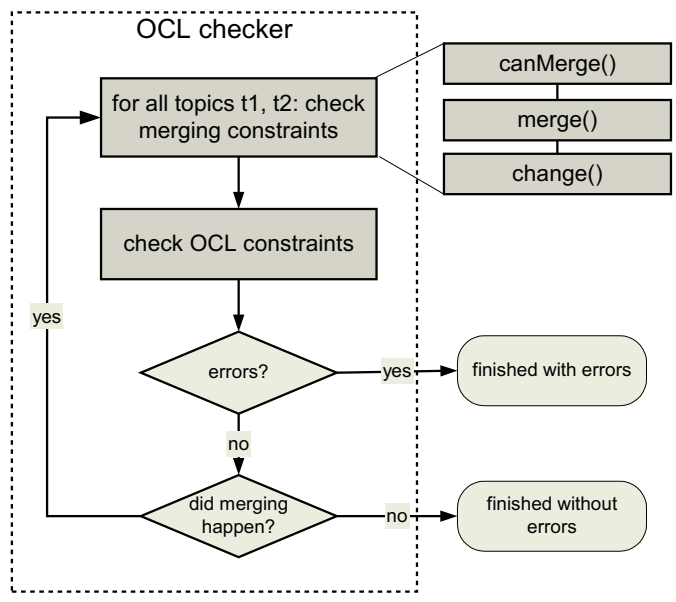

Fig. 7. Process Steps of the OCL Checker

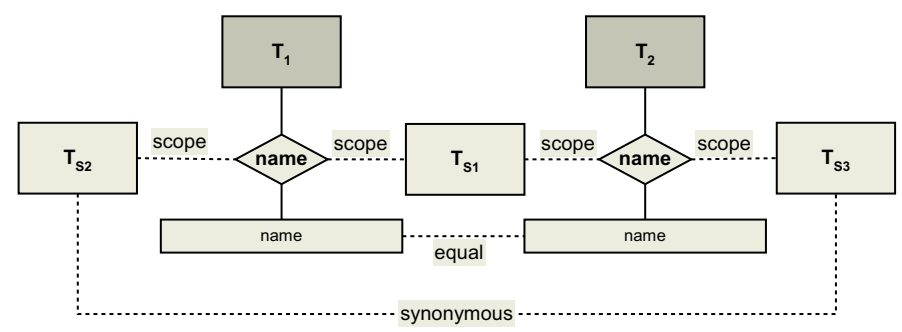

Fig. 8. One Merge Causing a Further Merge

There, $T_{1}$ has a name in the scope $\left\{T S_{1}, T S_{2}\right\}$, and $T_{2}$ has the same name in the scope $\left\{T S_{1}, T S_{3}\right\}$. In a first processing cycle of the OCL checker, $T_{1}$ and $T_{2}$ are considered to stand for different subjects and are therefore not merged. Suppose that $T S_{2}$ and $T S_{3}$ are merged (due to identifying the same subject). Then, $T_{1}$ and $T_{2}$ had the name in the same scope and could also be merged, but only in the second processing cycle of the OCL checker. These processing cycles have to be implemented by a fix-point algorithm, stopping if either an error occurred (a constraint was violated) or a complete cycle has run without performing a single merge. This is shown in fig. 7 .

\section{Conclusion}

In this paper, we have treated the merging of topics in XTM in detail. We have shown how the XTM merging conditions can be realized with the TMUML meta model and presented the according OCL constraints and operations. These operations and constraints can be tested straightforwardly with the USE tool 
[5], which helps creators of Topic Maps systems trying out different strategies and their implications. We identified further difficulties when merging different kinds of topics, including association types, occurrence types, topic types and topic instances. After presenting suggestions to overcome these difficulties using OCL, we sketched the processing steps of the OCL checker, which is the central component for validating the well-formedness of topic maps.

All in all, the more voluminous and extensive a Topic Maps system is designed to be, the more important is the issue of merging. At this point, we want to emphasize the duality of merging. On one hand, it provides a great gain in extending existent knowledge by additional facts and information. Two topics may be part of topic maps of different authors. When their associations, occurrences, names and types are combined due to merging, the resulting topic holds the knowledge about a subject of both of the authors. One may learn from the other, and the quality of search queries against topic maps as well as the navigational access on topic maps may enormously benefit from the combined information about a subject.

On the other hand, merging that is applied "silently", simply checking the existing conditions, may always cause subjectively undesired effects. Consider the case two authors both created a topic with the same name, but the second author added another name that the first author would not recognize as a valid name for the topic. No merging process could automatically detect and avoid such situations. Therefore, a notification system seems very important to be integrated with a Topic Maps repository. Every merging should be reported (e.g. by e-Mail, a newsletter system or at least a common logging file or database) to the originators of the merged topics, so that they can verify the semantic impacts of the merge and possibly correct undesired effects (or even manually take back the merge). Another strategy can be adding a special attribute, let us call it "mergeable", to each topic, providing a flag that may avoid merging the topic in any case, even if it would have to be merged according to XTM. The drawback would be that possibly merging would happen quite rarely, and it would be a proprietary extension to XTM.

Our further work will contain an explicit description of the transformation of XTM to a UML compliant, intermediate format like XMI [9] or the USE syntax. The implementation of this transformation together with the implementation of the OCL checker will make up a validator for well-formed XTM Topic Maps, where OCL constraints can dynamically be added or modified in the according USE file and need not be hard-coded into the OCL checker.

\section{References}

1. Ahmed, K.: Topic Maps for repositories. Proceedings XML Europe 2000. GCA, Paris (2000).

2. Auillans, P., de Mendez, P.O., Rosenstiehl, P., Vatant, B.: A formal model for Topic Maps. International Semantic Web Conference (ISWC) 2002.

http://www.mondeca.com/english/publications_-_doc.htm, last visited 23.2.2003 
3. Baird, C.: Topic Map Cartography - a discussion of Topic Map authoring. Proceedings XML Europe 2000. GCA, Paris (2000)

4. Gogolla, M., Richters, M.: On constraints and queries in UML. In: Schader, M., Korthaus, A. (eds.): The Unified Modeling Language - Technical Aspects and Applications, p. 109-121. Physica, Heidelberg (1998)

5. Gogolla, M., Richters, M.: Development of UML Descriptions with USE. In: Tjoa A.M., Shafazand, H., Badie K. (eds.): Proc. 1st Eurasian Conf. Information and Communication Technology (EURASIA'2002). LNCS, Springer Verlag, Berlin Heidelberg New York (2002)

6. Gronmo, G.O.: Creating semantically valid topic maps. Proceedings XML Europe 2000. GCA, Paris (2000)

7. ISO/IEC 13250: Information technology - SGML Applications - Topic Maps. International Organization for Standardization, Geneve, Switzerland (1999)

8. Mueck, T.A., Widhalm, R.: Schlagwort - Topic Maps. Wirtschaftsinformatik, 3:297300. Verlag Vieweg, Wiesbaden (2001)

9. Object Management Group: OMG UML Specification Version 1.3. http://www.omg.org./uml/, last visited 1. 8. 2002

10. Object Management Group: XMI XML Metadata Interchange, Version 1.1. http://cgi.omg.org/docs/ad/99-10-02.pdf, last visited 2. 8. 02

11. Ontopia: The Ontopia Schema Language Reference Specification, Version 1.3. http://www.ontopia.net/omnigator/docs/schema/spec.html, last visited 23. 10. 2002

12. Pepper, S., Moore, G. et al: XML Topic Maps (XTM) 1.0. Topic Maps Authoring Group (2001). http://www.topicmaps.org/xtm/1.0/, last visited 19. 6. 2001

13. Pepper, S.: Draft requirements, examples and a "low bar" proposal for Topic Map Constraint Language. ISO/IEC JTC 1/SC34/WG3 (2001).

http://www.y12.doe.gov/sgml/sc34/document/0226.htm, last visited 1. 8. 2002

14. Rath, H.H.: Technical Issues on Topic Maps. Proceedings MetaStructures 99. GCA, Alexandria, VA (1999)

15. IETF (Internet Engineering Task Force) RFC 2396: Uniform Resource Identifiers (URI): Generic Syntax. IETF (1998). http://www.ietf.org/rfc/rfc2396.txt, last visited 17. 1. 2003

16. Richters, M., Gogolla, M.: On formalizing the UML object constraint language OCL. In: Tok-Wand, L. (ed.): Proc. 17th Int. Conf. Conceptual Modeling (ER'98), p. 449-464. LNCS Vol. 1507, Springer Verlag, Berlin Heidelberg New York (1998)

17. Warmer, J., Kleppe, A.: The object constraint language - precise modeling with UML. Addison-Wesley (1999).

18. Widhalm, R., Mueck, T.A.: Topic Maps - Semantische Suche im Internet. Springer Verlag, Heidelberg (2002).

19. Widhalm, R., Mueck, T.A.: Web metadata semantics - on the road to well-formed topic maps. In: Ozsu T. et al. (eds.): Proc. 2nd Int. Conf. On Web Information Systems Engineering (WISE), vol. 2, p. 141-150. IEEE Computer Society, Los Alamitos, CA (2002).

20. Widhalm, R., Mueck, T.A.: Well-formed Topic Maps. Submitted to SoSyM, Journal on Software \& System Modeling. Springer Verlag, Berlin Heidelberg New York (2003) 